\title{
Asymmetric Cyanosilylation of Aldehydes by Chiral Ti-TADDOL Complex
}

\author{
Sung Soo Kim, ${ }^{*}$ Ju Myung Kwak, and Gurusamy Rajagopal' \\ Department of Chemistry, Inha University, Incheon 402-751, Korea. "E-mail: sungsoo@inha.ac.kr \\ Received Angust 3, 2006
}

\begin{abstract}
$\Lambda$ variety of chiral cyanohydrins were readily prepared from aldehydes with TMSCN under the influence of chiral titanium complex formed in situ from Ti( $\left.\mathrm{O}^{\prime} \mathrm{Pr}\right)_{4} / \mathrm{TADDOL}(\mathrm{I})$ in the presence of $\mathrm{Ph}_{3} \mathrm{PO}$ as additive. The double activation method produces trimethylsilylethers in excellent yield $(95 \%)$ with moderate enantiomeric excess.
\end{abstract}

Key Words : Cyanosilylation, $\Lambda$ ldehyde, Ti-TADDOL, Double activation

\section{Introduction}

Enantiomerically pure cyanohydrins are important building blocks for the synthesis of several 1,2-bifunctional products such as $\alpha$-hydroxy acids, $\alpha$-hydroxy amines, $\alpha$ hydroxy alcohols and $\alpha$-amino acid derivatives. ${ }^{2.3}$

A wide range of titanium complexes have been elaborated for organic transformations. ${ }^{+}$The chiral ligand for titanium based catalyst include BINOLs, tartrate esters, ${ }^{6}$ sulfoximes, peptides, ${ }^{8}$ Schiff bases ${ }^{9}$ and others. ${ }^{10}$ In each case complexation of the ligand to a suitable titanium salt generated a chiral complex that induced the asymmetric addition of hydrogen and /or trimethylsilylcyanation to aldehydes.

Shibasaki ${ }^{11}$ disclosed enantioselective catalytic addition of TMSCN to carbonyl compounds by using carbohydrate based ligands and $\mathrm{Ti}(\mathrm{OPr})_{4}$. Belokon and $\mathrm{North}^{12}$ have reported the asymmetric silylcyanation of carbonyl compounds. $\mathrm{Bu},{ }^{13}$ Zhou, ${ }^{14}$ Feng, ${ }^{15}$ and Gennari ${ }^{16}$ have reported trimethylsilylcyanation of carbonyl compounds by using modified and bulky salen ligand and $/ \operatorname{Ti}\left(\mathrm{O}^{\prime} \mathrm{Pr}\right)_{4}$. Recently we reported ${ }^{17}$ asymmetric addition of TMSCN to carbonyl compounds by using $\mathrm{Al}$ (salen) and $\mathrm{Mn}$ (salen) complexes.

To the best of our knowledge, TADDOLs originated by Seebach ${ }^{18}$ represented a versatile class of chiral auxiliaries or ligands in asymmetric synthesis. However no example of cyanosilylation of carbonyl compounds has been reported using TADDOL $/ \mathrm{Ti}\left(\mathrm{O}^{\prime} \mathrm{Pr}\right)_{4}$ system. Thus we are interested in the exposing the usability of this ligand in cyanosilylation reactions.

\section{Results and Discussion}

Cyanosilylation reaction was started with benzaldehyde as a test substrate. The results of these reactions are summarized in Table 1 . Various solvents including $\mathrm{CH}_{2} \mathrm{Cl}_{2}, \mathrm{THF}$, $\mathrm{CH}_{3} \mathrm{CN}$ and $\mathrm{CHCl}_{3}$ were examined among which $\mathrm{CHCl}_{3}$ was found to be the best solvent for this reaction at RT (Table 1, entries 1-4).

The enantioselectivity was greatly influenced by the reaction temperature. The best perfonnance was at $-10^{\circ} \mathrm{C}$, while at higher temperatures the enantioselectivity was poorly controlled (entries 5 and 6 ). The reaction was performed
Table 1. Cyanosilylation Benzaldehyde under various conditions

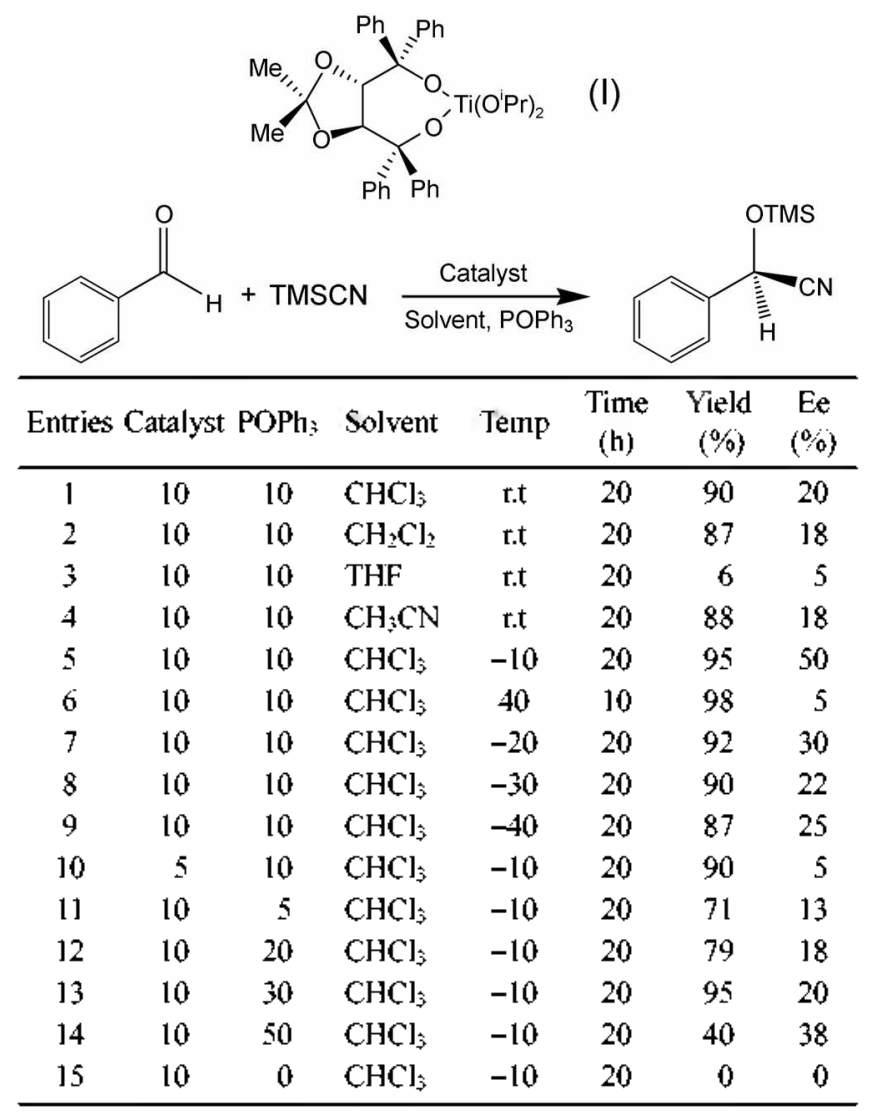

at very low temperatures $\left(-20^{\circ} \mathrm{C}\right.$ to $\left.-40^{\circ} \mathrm{C}\right)$, but decreased enantiomeric excesses were observed (entries 7, 8 and 9). Decreasing the catalytic loading affects the yield and e.e. negatively (entry 10). Additive quantity was varied (entries 5 and $11-14$ ) and $10 \mathrm{~mol} \%$ (entry 5) of triphenylphosphonium oxide proved to be optimal, without which no reaction took place (entry 15).

The reaction conditions in Entry 5 of the Table I were applied to the cyanosilylation reaction of various aldehydes with TMSCN (Table 2). Benzaldehydes with various substituents at para position were transformed into the corresponding chiral cyanohydrins (entries 1-7) with moderate enantioselectivity in over $95 \%$ yield. 
Table 2. Trimethylsilylcyanation of Nldehydes ${ }^{\text {ish }}$

Entry

"a $10 \mathrm{~mol} \%$ of 1 and $10 \mathrm{~mol} \%$ of $\mathrm{Ph}_{3} \mathrm{PO}{ }^{b} \mathrm{All}$ reactions were conducted at $-10^{\circ} \mathrm{C}$ for $20 \mathrm{~h}$. "All products are R configuration.

The e.e. obtained was good to moderate $(40-60 \%)$. Replacement of benzene ring by furan moiety gave little difference on the isolated yield (entry 8). Introduction of conjugate double bond with respect to carbonyl group hardly produced significant effect on enantioselectivity (entry 9). 3Phenylpropanal (entry 10) reacted smoothly with TMSCN to give the corresponding cyanohydrin. Based on our previous experience $^{17}$ in cyanosilylation reactions and related titanium based work ${ }^{18.19}$ the possible mechanism and transition state involved in the enantioselective reaction catalyzed by $\mathbf{I}$ is given in Figure 1 .

In conclusion highly efficient double activation catalysis by TADDOL/ Ti(O-Pr $)_{4}$ and $\mathrm{Ph}_{3} \mathrm{PO}$ has been developed for the enantioselective cyanosilylation of various aldehydes. The cyanosilylation reaction takes place under comparatively mild conditions in terms of temperature and reaction time.

\section{Experimental Section}

General method for the asymmetric addition of trimethylsilylcyanide to benzaldehyde: To a stirred solution of $(+) 4,5-$ bis[dihydroxy (diphenyl)-methy]] 2,2-dimethyl-1,3-dioxane

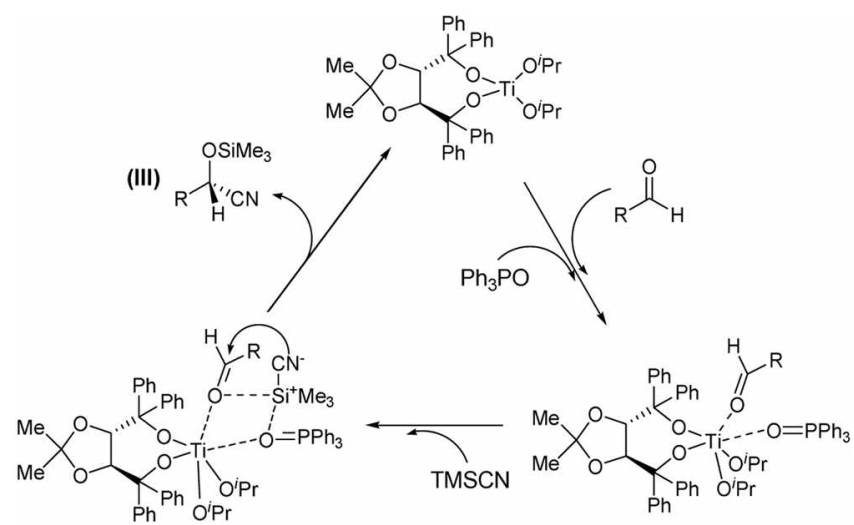

(II)

Figure 1

(TADDOL) $(10 \mathrm{~mol} \%$ ) in choloroform $(2 \mathrm{~mL})$ was added titanium tetraisopropoxide $(10 \mathrm{~mol} \%)$ under $\mathrm{N}_{2}$ at room temperature and the mixture was stirred for 1 hour. Trimethylsilyl cyanide ( $2 \mathrm{mmol}$ ) was added to the reaction mixture and stirred for an additional 0.5 hour. Then, the reaction mixture was cooled to $-10^{\circ} \mathrm{C}$ and benzalaldehyde (1 mmol) was added to the reaction mixture. The disappearance of the aldehyde was monitored by thin layer chromatography. After completion of the reaction $\mathrm{CHCl}_{3}$ was evaporated. The crude product was purified by flash chromatography (hexane: ethyl acetate $=9: 1$ ).

2-Hydroxy-2-phenylacetonitrile. ' $\mathrm{H}$ NMR $\left(\mathrm{CDCl}_{3}, 200\right.$ $\mathrm{MHz}): \delta 7.44(\mathrm{~m}, 5 \mathrm{H}), 6.15(\mathrm{~s}, \mathrm{lH}), 4.22(\mathrm{~m}, 2 \mathrm{H}), 1.27(\mathrm{t}, J$ $=7.0 \mathrm{~Hz}, 3 \mathrm{H}){ }^{13} \mathrm{C} \mathrm{NMR}\left(\mathrm{CDCl}_{3}, 50 \mathrm{MHz}\right): \delta 153.6,131.3$, $130.8,129.4,127.9,115.6,66.3,65.6,14.3$ HRMS $\left(\mathrm{M}^{+}\right)$ cacld for $\mathrm{C}_{1} \mathrm{H}_{11} \mathrm{NO}_{3} 205.0739$ found $205.0753 \mathrm{R}$ enantiomer in 50\% ee. HPLC (DAICEL CHIRALCEL OD) 11.5 $\mathrm{min}$ and $14.0 \mathrm{~min}$ (The enantiomeric excess was determined by HPLC after conversion to ethyl carbonate).

2-Hydroxy-2-(4-methyphenyl)acetonitrile. ' $\mathrm{H}$ NMR $\left(\mathrm{CDCl}_{3}, 200 \mathrm{MHz}\right): \delta 7.44(\mathrm{~d}, J=8.25 \mathrm{~Hz}, 2 \mathrm{H}), 7.25$ (d, $J=$ $8.25 \mathrm{~Hz}, 2 \mathrm{H}), 6.22(\mathrm{~s}, 1 \mathrm{H}), 4.28(\mathrm{~m}, 2 \mathrm{H}), 2.39(\mathrm{~s}, 3 \mathrm{H}), 1.35$ $(\mathrm{t}, J=7.3 \mathrm{~Hz}, 3 \mathrm{H}){ }^{13} \mathrm{C} \mathrm{NMR}\left(\mathrm{CDCl}_{3}, 50 \mathrm{MHz}\right): \delta 153.5$, $140.7,129.9,128.6,127.9,115.9,66.2,65.3,21.4,14.1$ HRMS $\left(\mathrm{M}^{+}\right)$cacld for $\mathrm{C}_{12} \mathrm{H}_{13} \mathrm{NO}_{3} 219.0895$ found 219.0889 $\mathrm{R}$ entiomer in $57 \%$ ee. HPLC (DAICEL CHIRALCEL OD) $17.4 \mathrm{~min}$ and $19.5 \mathrm{~min}$ (The enantiomeric excess was determined by HPLC after conversion to ethyl carbonate).

2-Hydroxy-2-(4-methoxy phenyl) acetonitrile. ' $\mathrm{H}$ NMR $\left(\mathrm{CDCl}_{3}, 200 \mathrm{MHz}\right): \delta 2.8$ (brs, $\left.1 \mathrm{H}\right), 3.86(\mathrm{~s}, 3 \mathrm{H}), 5.48(\mathrm{~s}$, $1 \mathrm{H}), 6.95(\mathrm{~d}, J=8.5 \mathrm{~Hz}, 2 \mathrm{H}), 7.46(\mathrm{~d}, J=8.5 \mathrm{~Hz}, 2 \mathrm{H}){ }^{13} \mathrm{C}$ $\mathrm{NMR}\left(\mathrm{CDCl}_{3}, 50 \mathrm{MHz}\right): \delta 55.40,63.27,114.52,118.93$, $127.50,128.29,160.70 \mathrm{R}$ enantiomer in $40 \%$ ee. HPLC (DAICEL CHIRALCEL OD) $14.8 \mathrm{~min}$ and $12.7 \mathrm{~min}$ (The enantiomeric excess was determined by HPLC after conversion to the corresponding acetyl ester).

2-Hydroxy-2-(4-tert-butylphenyl) acetonitrile. ${ }^{1} \mathrm{H}$ NMR $\left(\mathrm{CDCl}_{3}, 200 \mathrm{MHz}\right): \delta \mathrm{l} .25(\mathrm{~s}, 9 \mathrm{H}), 2.88(\mathrm{~s}, 1 \mathrm{H}), 5.43(\mathrm{~s}, \mathrm{IH})$, $7.37(\mathrm{~m}, 4 \mathrm{H}){ }^{13} \mathrm{C} \mathrm{NMR}\left(\mathrm{CDCl}_{3}, 50 \mathrm{MHz}\right): \delta 31.64,35.18$, $63.77,119.46,126.56,126.94,132.79,153.56 \mathrm{HRMS}\left(\mathrm{M}^{+}\right)$ cacld for $\mathrm{C}_{12} \mathrm{H}_{15} \mathrm{NO} 189.1154$ found $189.1143 \mathrm{R}$ enantiomer 
in $60 \%$ ee. HPLC (DAICEL CHIRALCEL OD) $21.5 \mathrm{~min}$ and $24.0 \mathrm{~min}$.

2-(3-fluoro-4-methoxyphenyl)-2-(trimethylsilyloxy) acetonitrile. ${ }^{1} \mathrm{H} \mathrm{NMR}\left(\mathrm{CDCl}_{3}, 200 \mathrm{MHz}\right): \delta 2.2$ (brs, $\left.1 \mathrm{H}\right), 3.83$ (s, $3 \mathrm{H}), 5.48(\mathrm{~s}, 1 \mathrm{H}), 6.95(\mathrm{~d}, J=8.5 \mathrm{~Hz}, 2 \mathrm{H}), 7.06(\mathrm{~s}, 1 \mathrm{H}){ }^{13} \mathrm{C}$ $\mathrm{NMR}\left(\mathrm{CDCl}_{3}, 50 \mathrm{MHz}\right): \delta 55.80,65.27,115.52,116.93$, $118.50,127.29,147.70,151.3 \mathrm{R}$ enantiomer in $42 \%$ ee. HPLC (DAICEL CHIRALCEL OD) $12.8 \mathrm{~min}$ and $14.7 \mathrm{~min}$.

2-(naphthalen-2-yl)-2-(trimethylsilyloxy) acetonitrile. 'H NMR ( $\left.\mathrm{CDCl}_{3}, 200 \mathrm{MHz}\right): \delta 8.01(\mathrm{~m}, 3 \mathrm{H}), 7.55(\mathrm{~m}, 3 \mathrm{H})$, $7.17(\mathrm{~m}, 1 \mathrm{H}), 5.02(\mathrm{~s}, 1 \mathrm{H}),{ }^{13} \mathrm{C} \mathrm{NMR}\left(\mathrm{CDCl}_{3}, 50 \mathrm{MHz}\right): \delta$ $132.81,128.9,128.08,127.63,126.77,126.58,125.6,123.5$, $119.0,63.795 \mathrm{R}$ enantiomer in $52 \%$ ee. HPLC (DAICEL CHIRALCEL OD) $15.5 \mathrm{~min}$ and $18.0 \mathrm{~min}$.

2-Hydroxy-2-(3-phenoxyphenyl) acetonitrile. ${ }^{1} \mathrm{H}$ NMR $\left(\mathrm{CDCl}_{3}, 200 \mathrm{MHz}\right): \delta 3.5$ (brs, $\left.1 \mathrm{H}\right), 5.46(\mathrm{~s}, 1 \mathrm{H}), 7.5(\mathrm{~m}, 9 \mathrm{H})$ ${ }^{13} \mathrm{C} \mathrm{NMR}\left(\mathrm{CDCl}_{3}, 50 \mathrm{MHz}\right): \delta 63.17,116.63,118.59,119.65$, $120.94,123.94,129.93,130.52,137.05,156.34,158.12 \mathrm{R}$ entiomer in $40 \%$ ee. HPLC (DAICEL CHIRALCEL OD) $28.1 \mathrm{~min}$ and $40.9 \mathrm{~min}$ (The enantiomeric excess was determined by HPLC after conversion to the corresponding acetyl ester).

2-(2-Furyl)-2-hydroxyethanenitrile. ${ }^{1} \mathrm{H}$ NMR $\left(\mathrm{CDCl}_{3}\right.$, $200 \mathrm{MHz}): \delta 7.50(\mathrm{dt}, J=1.8 \mathrm{~Hz}, 1 \mathrm{H}), 6.65(\mathrm{~d}, J=3.4 \mathrm{~Hz}$, $1 \mathrm{H}), 6.44(\mathrm{~s}, 1 \mathrm{H}), 6.47(\mathrm{dd}, J=3.4,1.8 \mathrm{~Hz}, 1 \mathrm{H}), 2.15(\mathrm{~s}, 3 \mathrm{H})$ ${ }^{13} \mathrm{C} \mathrm{NMR}\left(\mathrm{CDCl}_{3}, 50 \mathrm{MHz}\right): \delta-0.43,57.41,109.70,110.78$, $117.11,143.84,148.20 \mathrm{R}$ entiomer in $40 \%$ ee. HPLC (DAICEL CHIRALCEL AS) $8.7 \mathrm{~min}$ and $9.7 \mathrm{~min}$ (The enantiomeric excess was detemined by HPLC after conversion to the corresponding acetyl ester).

2-Hydroxy-4-phenyl-3-butenenitrile. ${ }^{1} \mathrm{H}$ NMR $\left(\mathrm{CDCl}_{3}\right.$, $200 \mathrm{MHz}): \delta 7.43(\mathrm{~m}, 5 \mathrm{H}), 6.98(\mathrm{~d}, J=15.9 \mathrm{~Hz}, 1 \mathrm{H}), 6.19$ (dd, $J=15.9,6.7 \mathrm{~Hz}, 1 \mathrm{H}), 6.03(\mathrm{dd}, J=6.7,0.9 \mathrm{~Hz}), 2.15$ (s, $3 \mathrm{H}){ }^{13} \mathrm{C} \mathrm{NMR}\left(\mathrm{CDCl}_{3}, 50 \mathrm{MHz}\right): \delta 168.9,137.9,134.6$, $129.6,128.9,127.1,118.4,115.7,61.5,20.5$ HRMS $\left(\mathrm{M}^{+}\right)$ cacld for $\mathrm{C}_{12} \mathrm{H}_{11} \mathrm{NO}_{2} 201.0790$ found $201.0782 \mathrm{R}$ entiomer in $44 \%$ ee. HPLC (DAICEL CHIRALCEL OD) $18.1 \mathrm{~min}$ and $22.3 \mathrm{~min}$ (The enantiomeric excess was determined by HPLC after conversion to the corresponding acetyl ester).

2-Hydroxy-4-phenylbutanenitrile. ${ }^{1} \mathrm{H}$ NMR ( $\mathrm{CDCl}_{3}$, $200 \mathrm{MHz}): \delta 7.31(\mathrm{~m}, 2 \mathrm{H}), 7.22(\mathrm{~m}, 3 \mathrm{H}), 4.44(\mathrm{t}, J=6.4 \mathrm{~Hz}$, $1 \mathrm{H}), 2.80(\mathrm{~m}, 2 \mathrm{H}), 2.15(\mathrm{~m}, 2 \mathrm{H}), 0.92(\mathrm{~s}, 9 \mathrm{H}), 0.18(\mathrm{~s}, 3 \mathrm{H})$, $0.12(\mathrm{~s}, 3 \mathrm{H}){ }^{13} \mathrm{C} \mathrm{NMR}\left(\mathrm{CDCl}_{3}, 50 \mathrm{MHz}\right): \delta 140.0,128.5$, $128.4,126.2,119.9,61.3,37.9,25.8,18.1,20.51,25.3 \mathrm{R}$ entiomer in $59 \%$ ee. HPLC (DAICEL CHIRALCEL OD) $8.7 \mathrm{~min}$ and $10.9 \mathrm{~min}$.

Acknowledgements. We warmly thank The Center for Biological Modulators for the financial support. One of us
(GR.) thanks the Management, Correspondent, Principal and HOD, Chemistry of B.S.A. Crescent Engineering College, Chennai, India for permitting him to do PDF research.

\section{References}

I. Dr. G. Rajagopal is a Brain Pool Scientist from Dept of Chemistry, BSA Crescent Engineering College, Chennai 600048 , India and he was assisted by a grant from the Korean Federation of Science and Technology Societies and Korea Science and Engineering Foundation.

2. Ojima, I. Catalytic Asymmetric Symthesis, 2nd ed.; Wiley-VCH; 2000.

3. (a) Gregory, R. J. H. Chem. Rev 1999, 99, 3649. (b) Brunel, J. M.; Holmes, I. P. Angen. Chem. Int. Ed. Engl. 2004, 43, 2752. (c) Kurono, N.; Yamaguchi, M.; Suzuki, K.; Ohkuma, T. J. Org. Chem. 2005, 70, 6530. (d) Liu, X.; Qin, B.; Zhou, X.; He, B.; Feng, X. J. Am. Chem, Soc. 2005, 127, 12224. (e) Itazaki, M.; Nakazawa, H. Chent. Lett. 2005, 34, 1054. (f) Iwanami, K.; Oriyama, T. Chem. Letf. 2004, 33, 132.

4. Ramo'n, D. J.; Yus, M. Chent. Rev: 2006, 106, 2126.

5. Mori, M.; Imma, H.; Nakai, T. Tetrahedron Lett. 1997, 38, 6229.

6. Hayashi, M.; Matsuda, T.; Oguni, N. J. Chem, Soc. Chem. Commun, 1990, 1364.

7. North, M. Tetrahedron: Asymmetry 2003, 14, 147.

8. Nitta, H.; Yu, D.; Kudo, M.; Mori, A.; Inoue, S. J. Am. Chem. Soc. 1992, 114,7969 .

9. North, M. Synlett 1993, 807.

10. Yang, Z.; Zhou, Z.; He, K.; Wang, L.; Zhao, G.; Zhou, Q.; Tang, C. Tetrahedron: Asynmetry 2003, 14, 3937.

11. (a) Hanashima, Y.; Kanai, M.; Shibasaki, M. J. Am. Chem. Soc. 2000, 122, 7412. (b) Hanashima, Y.; Kanai, M.; Shibasaki, M. Tetrahedron Lett. 2001, 42,69l.

12. (a) Belokon, Y.; Green, B.; Ikonnikov, V.; North, M.; Parsons, T.; Tararov, V. I. Tetrahedron 2001, 5, 77I. (b) Belokon, Y. N.; Blacker, A. J.; Carta, P.; Clutlerbuck, L. A.; North, M. Tetrahedron 2004, 60, 10433.

13. Liang. S.; Bu, X. R.J. Org. Chem. 2002, 67, 2702.

14. He, K.; Zhou, Z.; Wang, L.; Li, K.; Zha, G; Zhou, Q.; Tang, C. Symleft 2004, 1521 .

15. Li, Y.; He, B.; Qin, B.; Feng, X.; Zhang, G. J. Org. Chem. 2004, 69,7910 .

16. Steele, R. M.; Monti, C.; Gennari, C.; Piarulli, U.; Andreoli, F.; Vanthuyne, N.; Roussel, C. Tetahedron: Asymmetry 2006, 17 , 999.

17. (a) Kim, S. S.; Lee, S. H. Synth. Conmtm. 2005, 35, 751. (b) Kim, S. S.; Song, D. H. Ezr: J. Org. Chem, 2005, 1777, (c) Kim, S. S.; Kwak, J. M. Tetrahedron 2006, 62, 49. (d) Kim, S. S. Pure Appl. Chem. 2006, 78, 977. (e) Kim, \$. S.; Lee, S. H.; Kwak, J. M. Tetrahedron:Asymintetry 2006, $17,1165$.

18. Seebach, D.; Beck, A. K.; Heckel, A. Angew. Chem. Int. Ed. 2001, 40,92 .

19. (a) Uang, B. J.; Fu, I. P.; Hwang, C. D.; Chang, C. W.; Yang, C. T.; Hwang, D. R. Tetrahedron 2004, 60, 10479. (b) Hayashi, M.; Miyamoto, Y.; Inoue, T.; Oguni, N. J. Org. Chen. 1993, 58, 1515. (c) Jiang, Y.; Zhou, X. H.; Wenhao, W.; Mi, A. L. Tetrahedron:Asynimetry 1995, 6, 405. 